\title{
Obituaries / Nachrufe / Nécrologie / Necrologia
}

\section{Daniel Bovet}

1907-1992

In Rom verstarb im Alter von 85 Jahren Daniel Bovet, der fünfte schweizerische Nobelpreisträger für Medizin und Physiologie (1957, nach Th. Kocher, P. Müller, W. R. Hess, T. Reichstein, vor W. Arber). Geboren in Neuenburg als Sohn des Psychologen Pierre Bovet, studierte Bovet in Genf Biologie und assistierte beim Physiologen Battelli. 1929 begann seine überaus erfolgreiche Laufbahn als Pharmakologe, zuerst als Assistent und später als Nachfolger von Fourneau am Institut Pasteur in Paris. Nach dem Zweiten Weltkrieg gründete und leitete er das Laboratorio di Chimica Terapeutica am Istituto Superiore di Sanità in Rom. Nach einem Zwischenspiel als Lehrstuhlinhaber für Pharmakologie an der Universität Sassari kehrte er nach Rom zurück, wo er bis 1976 ein Laboratorium für Psychobiologie leitete und eine Professur bekleidete.

Bovets Aktivitäten und Leistungen lassen sich den folgenden fünf Themenkreisen zuordnen :

- Entdeckung der ersten synthetischen sympathikolytischen Substanzen, welche den ersten Schritt zu den späteren Antihypertensiva bildeten.

- Entdeckung der ersten Antihistaminica als Mittel gegen Allergien, Reisekrankheit und Erbrechen.

- Entwicklung der curare-ähnlichen Muskelrelaxantien als wertvolle Bereicherung der Anästhesiologie.

- Entdeckung des wirksamen Prinzips von Domagks Prontosil und Eröffnung der Ära der Sulfonamide.

- Tierpsychologische und ethologische Methoden zum Verständnis zentralnervöser Leistungen wie Gedächtnis und Lernen.

Bovet hat die Pharmakologie durch die Anwendung einer chemischen Betrachtungsweise und durch systematische Ausarbeitung der Beziehungen zwischen Struktur und Aktivität von einem Anhang der Physiologie zu einer selbständigen Wissenschaft gemacht. Mit den Sulfonamiden wurde eine breite antibakterielle Chemotherapie möglich. Damit, und mit allen weiteren von ihm geschaffenen Mitteln, hatte er einen wesentlichen Anteil daran, dass die Medizin endlich auch in ein eigentliches therapeutisches Stadium eintrat. 
Bovets Beiträge sind in etwa 400 Publikationen festgehalten. Neben dem Nobelpreis ist er mit zahlreichen weiteren Ehrungen bedacht worden, blieb jedoch zu allen Zeiten ein Mensch der Bescheidenheit, der Freundlichkeit und der Güte. Ihm war auch bewusst, dass wissenschaftliche Fortschritte nicht gleichzusetzen sind mit Fortschritten der Medizin und der medizinischen Versorgung, da diese in hohem Masse von sozialen, politischen und wirtschaftlichen Bedingungen abhängig sind. Schliesslich galten Bovets Interessen auch der Geschichte seines Fachs, welche er durch eigene Beiträge bereichert hat.

Marcel H. Bickel 